\title{
INSIGHT INTO ATMOSPHERES OF EXTRASOLAR PLANETS THROUGH PLASMA PROCESSES
}

\author{
Ch. Helling*, and I. Vorgul*
}

\begin{abstract}
Extrasolar planets appear in a chemical diversity unseen in our own solar system. Despite their atmospheres being cold, continuous and transient plasma processes do affect these atmosphere where clouds form with great efficiency. Clouds can be very dynamic due to winds for example in highly irradiated planets like HD 189733b, and lightning may emerge. Lightning, and discharge events in general, leave spectral fingerprints, for example due to the formation of HCN. During the interaction, lightning or other flash-ionization events also change the electromagnetic field of a coherent, high energy emission, which results in a characteristic damping of the initial, unperturbed (e.g. cyclotron emission) radiation beam. We summarize this as 'recipe for observers'. External ionization by X-ray or UV e.g. from within the interstellar medium or from a white dwarf companion will introduce additional ionization leading to the formation of a chromosphere. Signatures of plasma processes therefore allow for an alternative way to study atmospheres of extrasolar planets and brown dwarfs.
\end{abstract}

\section{Introduction}

Extrasolar planets are discovered at an increasing rate and in an unexpected diversity (Figure 1). Now, observational efforts move towards analyzing these planets across the whole electromagnetic spectrum. Optical and near-infrared observations begin to provide insights into the chemical composition of exoplanetary atmospheres. Radio observations will allow to study the plasma processes involved. Though radio emission is typically observed from hot stellar plasma in astrophysics, any source of electrons accelerated in a magnetic field will create radio emission. Hallinan et al. [2008] and also Williams et al. [2015] demonstrated that radio emission emerges from brown dwarfs which have similar cool temperatures like giant gas planets (e.g. Jupiter, Saturn, HD 189733b). Based on these observations, brown dwarfs are suggested to have very strong magnetic fields of the

${ }^{*}$ Centre for Exoplanet Science, University of St Andrews, UK 
order of 1000 G. Hallinan et al. [2015] proposed that the radio emission observed on the late M-dwarf LSR J1835 + 3259 could be caused by a super-aurora. The dissipated energy is $10^{4}$ times larger than that produced in the Jovian magnetosphere, and a magnetic field strength of $1550 \mathrm{G} \ldots 2850 \mathrm{G}$ is inferred.

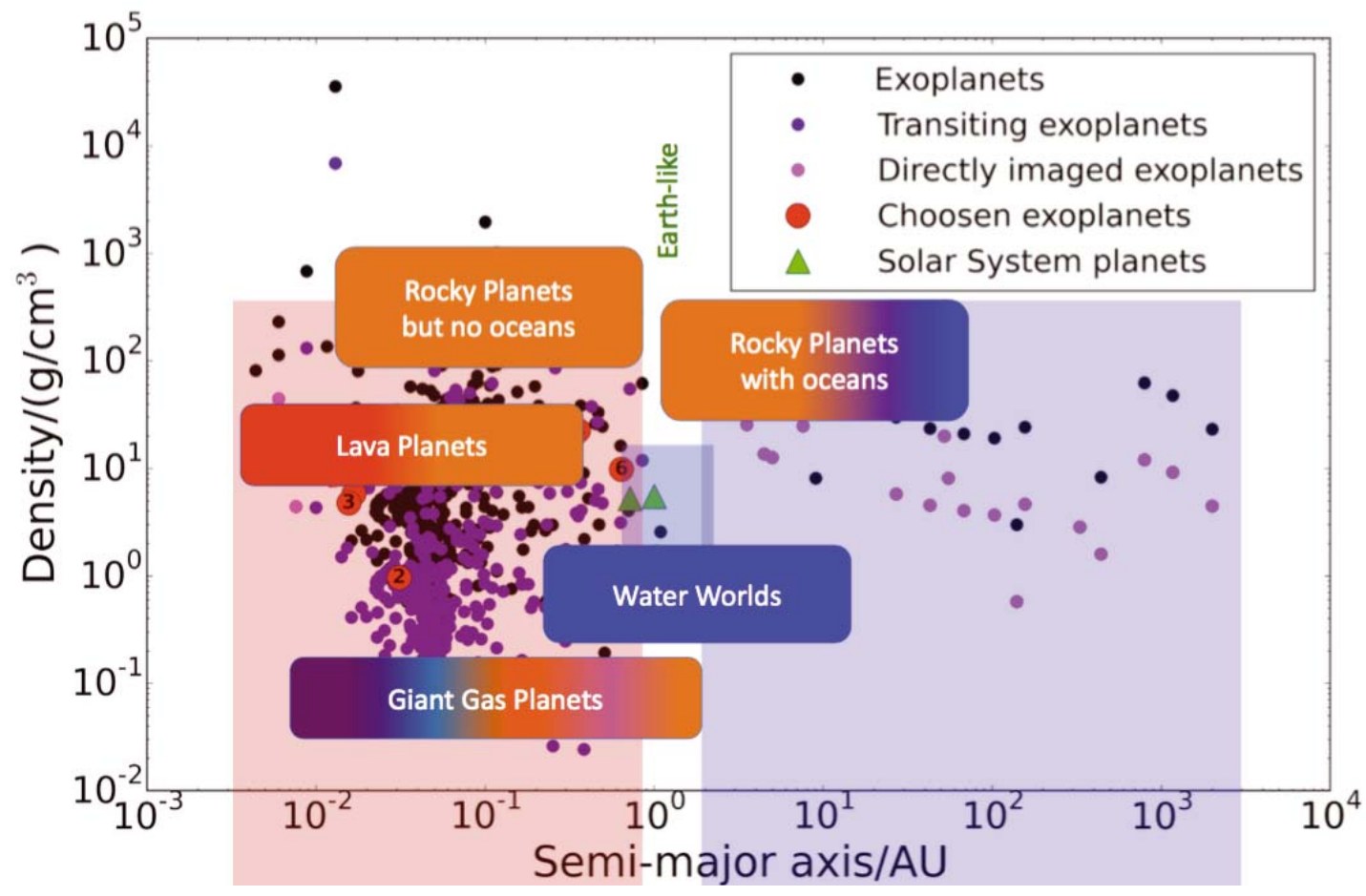

Figure 1: More than 3000 extrasolar planets are known. Bulk densities, as in this plot, show that their diversity reaches from Giant Gas Planets (low bulk density) to lava planets, water worlds, or rocky planets with or without oceans (high bulk density). The transit method allows to discover planets that are very near to their host star while the direct imaging method has shown that extrasolar planets can also exists rather far away from their host star. This diversity will also be reflected by the planet's atmosphere.

The emergence and the efficiency of plasma processes inside atmospheres is determined by the local degree of ionization which directly determines the atmospheric electric conductivity. The local degree of ionization is affected by the local thermal temperature, and by other atmospheric processes like strong winds (Alfvén ionization) or lightning. External cosmic ray or X-ray irradiation will affect the gas ionization, too, and hence link local plasma processes inside the atmosphere to environmental effects that are determined by the planet's host star and planetary system environment [e.g. Helling et al., 2016a,b; Longstaff et al., 2017]. Transient events (lightning), and continuous processes (external irradiation) can occur. Plasma processes can furthermore affect the local chemistry (e.g. lightning is natural source for $\mathrm{NO}_{\mathrm{x}}$ on Earth), and hence, they may appear as spectral fingerprints in the observed optical and near-IR spectra [e.g. Rimmer et al. 2014; Ardaseva et al., 2017]. A possible spectral fingerprint for lightning on extrasolar planets may be HCN [Hodosán et al., 2016]. 
Space- and ground-based observations [e.g. Sing et al., 2016; Nikolov et al., 2016] demonstrate that brown dwarfs and extrasolar planets are covered by clouds. Kinetic cloud formation models had predicted the formation of cloud particles with a wide variety of sizes and made of a mix of materials in brown dwarfs and extrasolar planets [Helling et al., 2008]. Lee et al. [2016] included the kinetic cloud modeling into a globally circulating atmosphere for HD 189733b and demonstrated the chemical diversity of clouds across the planetary globe. These simulations demonstrate that the atmosphere of the highly irradiated giant gas planet HD 189733b is filled with mineral cloud particles that either are small enough to follow the hydrodynamic wind motion due to frictional coupling, or cloud particles are big enough to frictionally decouple and fall into deeper atmospheric layers where their growth by chemical surface reactions is amplified due to higher densities (hence increased collision rates with the gas). Both cases suggest that cloud particles are charged due to triboelectric processes. Winds and gravitational settling provide mechanisms for large-scale charge separation, which then can lead to the emergence of lightning in these extrasolar atmospheres [see Hodosán et al., 2017].

\section{Magnetic coupling in weakly ionized atmospheres}

The collisionally dominated part of a brown dwarf/ giant gas planet atmosphere is rather cold for yielding a substantial degree, $f_{\mathrm{c}}$, of thermal ionization. The maximum values of $f_{\mathrm{c}}=p_{\mathrm{e}} / p_{\text {gas }} \approx 10^{-7}$ ( $p_{\mathrm{e}}$ : electron pressure, $p_{\text {gas }}$ : total gas pressure) occur at the top and bottom of the atmosphere [Rodriguez-Barrera et al., 2015]. The main electron donors are $\mathrm{K}^{+}$and $\mathrm{Na}^{+}$, followed by $\mathrm{Mg}^{+}$and $\mathrm{Fe}^{+}$.

Rodriguez-Barrera et al. [2015] used the cyclotron and the collisional frequencies to derive a maximum local magnetic field strength that would be required to achieve magnetic coupling of the local gas with $T_{\mathrm{e}}=T_{\mathrm{g} a s}$,

$$
B_{\mathrm{e}} \gg\left(m_{\mathrm{e}} / c\right) \sigma_{\text {gas }} n_{\text {gas }}\left[\left(k_{\mathrm{B}} T_{\mathrm{e}}\right) /\left(m_{\mathrm{e}}\right)\right]^{1 / 2}
$$

( $T_{\mathrm{e}}$ : electron temperature, $T_{\text {gas }}$ : gas temperature, $m_{\mathrm{e}}$ : electron mass, $c$ : speed of light, $\sigma_{\text {gas }}$ : collisional cross-section, $n_{\text {gas }}$ : gas number density, $k_{\mathrm{B}}$ : Boltzmann constant). The values of $B_{\mathrm{e}}$ indicate where the charged particle's motion will be dictated by an ambient magnetic field. Figure 2 demonstrates that the upper parts of brown dwarf and giant planet atmospheres can be magnetically coupled. The critical magnetic field strength is higher for ions than for electrons as shown in Figure 2.

Our findings suggest that non-irradiated brown dwarfs (and their giant gas planet counterparts) can develop a chromosphere as result of magnetic coupling of the upper atmosphere that will allow for e.g. Alfvén wave heating. Systematic $\mathrm{H} \alpha$ observations as presented by Schmidt et al. [2015] support this idea. We note that a chromosphere on brown dwarfs may not necessarily form from wave heating alone, but that external irradiation in form of high-energy X-ray in the ISM (interstellar medium), from a white-dwarf companion or inside a star-forming region may ionize the upper atmosphere completely [RodriguezBarrera, 2016]. This becomes of particular interest for white-dwarf/brown Dwarf binaries like the irradiated brown dwarf WD0137-349B [Casewell et al., 2015; Longstaff et al., 


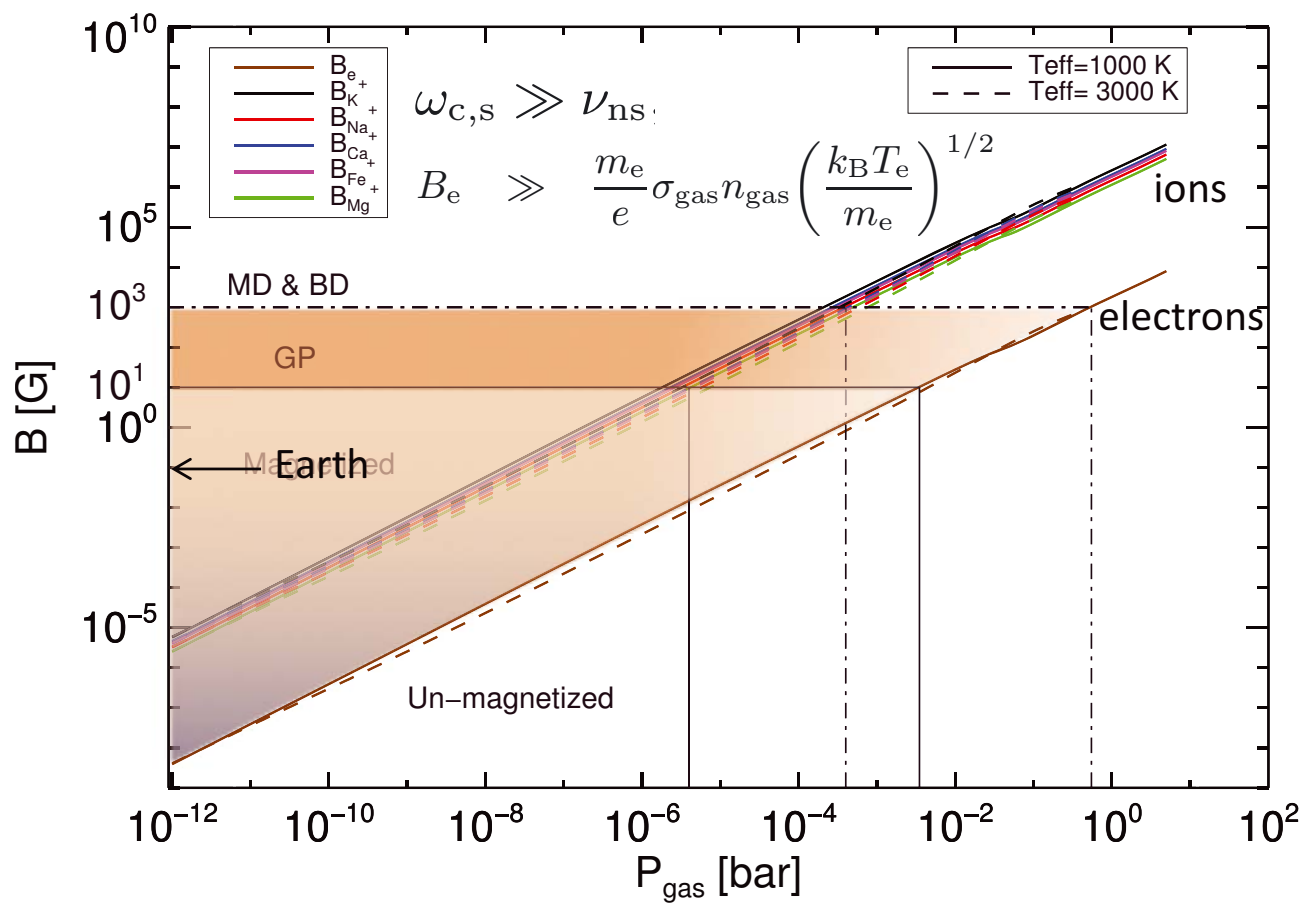

Figure 2: The critical magnetic field strength, $B_{e}[G]$ above which charged particle's motion will be dictated by an ambient magnetic field. The plot demonstrates that the upper atmosphere of objects with $T_{\text {eff }}=1000 \mathrm{~K}$ (solid lines) and $T_{\text {eff }}=3000 \mathrm{~K}$ (dashed lines) can be magnetically coupled already in the case of thermal ionization. Horizontal lines indicate boundaries for the shaded areas for $M$-dwarf/Brown Dwarfs and ( $M D \& B D$ ) and giant gas planets (GP). The closeness of the color lines shows that the specifics of the electron donors do not change the results significantly.

2017]. The high-energy radiation field of the white-dwarf primary does ionize the upper atmosphere of the companion brown dwarf which has been observed to emit in $\mathrm{He}, \mathrm{Na}$, $\mathrm{Mg}, \mathrm{Si}, \mathrm{K}, \mathrm{Ca}$, Ti and Fe. Consequently, the authors suggest that this brown dwarf most likely exhibits a chromosphere.

\section{The fingerprint of lightning in a coherent emission beam}

Brown dwarfs are observed to emit coherent radio emission which was interpreted as cyclotron maser emission [Hallinan et al., 2006]. The cyclotron radiation emerges nearly perpendicular to the magnetic field lines, but it will be refracted by the atmospheric material. The refracted radio beam may then cross atmospheric regions where lightning occurs (orange arrows in Figure 3), and the lightning will affect the beam's electromagnetic field such that it leaves a time-signature on it [Vorgul and Helling, 2016]. The geometry of this is shown in Figure 3. Examples for the different emission cone cases are discussed in Vorgul and Helling [2016], including CU Virgines showing a two-beam crossing due to the refraction of the atmosphere (cones 2), Neptune with $\gamma=80^{\circ}$ and $\Delta d=15^{\circ}$ (e.g. cone 3) or the Brown Dwarf NLTT 33370AB with $\gamma=35^{\circ}$ and $\Delta d=20^{\circ}-23^{\circ}$ (e.g. cone $4)$. 
Cyclotron maser emission (CME) can occur at relatively low plasma density only, when the electron plasma frequency is significantly smaller than the electron cyclotron frequency. Terrestrial CME originates from elongated plasma cavities along magnetic field lines [Pritchett et al., 2002; Burinskaya and Shevelev, 2017]. The source regions known as auroral cavities are of relatively low density compared to their surroundings. This low density allows the plasma frequency for the electrons to be much smaller than their cyclotron frequency. Figures 3 and 11 in Rodriguez-Barrera et al. [2015] suggest that this is the case also in brown dwarf and giant gas planet atmospheres. The emission from such cavities escapes due to gradual inhomogeneity of the surrounding plasma, for which a connecting branch between the X-mode (at which the emission is generated) and a vacuum propagating mode appears in dispersion curves [Cairns et al., 2008]. Expanding their consideration of the local dispersion relation, Cairns et al. [2011] show how global growing eigenmodes can be constructed for that scenario.

The cyclotron emission produces cavities that extend from $1000 \mathrm{~km}$ to $8000 \mathrm{~km}$ altitude in Earth's magnetosphere, but the most powerful emission occurs near the low altitude limit [Gray, 2012]. We suggest to probe a lightning-affected region with such a cyclotron emission. While most of the ground-to-cloud lightning discharges are initiated below the altitude at which CME originates, the interaction does not require the radiation crossing exactly the lightning channel, but the larger lightning-affected regions. Mallios and Pasko [2012] investigate charge transfer before, during and after a cloud-to-ground lightning discharge. The charges are transferred both from the cloud to the ground and from the cloud to the ionosphere. Mallios and Pasko [2012] show that the amount of charge that is transferred to the ground, due to currents flowing in the vicinity of the thundercloud during a transient time period following a lightning discharge (cloud-to-ground), can be several times smaller than the amount of charge that is transferred to the ionosphere during the same time period. The high amount of charges can then be transferred to an Earth altitude from $2 \mathrm{~km}$ to $13 \mathrm{~km}$, hence producing a quick rise of conductivity far away from the initial event. These are altitudes well matching those where the cyclotron emission occurring on Earth at the low altitude limit can propagate through, and so this sudden change in conductivity can be probed. It is then likely by analogy, that similar scenarios occur on other planets and also on brown dwarfs. Cyclotron radio signals from BDs come widened (large $\Delta d$ in Figure 3) compared to those from stars, which suggests that the emission is disturbed by propagation effects. This is likely to occur when CME is emitted in cavities and then escapes through an inhomogeneous plasma. Thus, we suggest to expect that cyclotron emission can probe the high-gradient conductivity rise due to lightning discharges. We point out that this idea of lightning affecting radio waves originating from other processes or sources, is not entirely new as e.g. Haldoupis et al. [2004], Inan et al. [2010] and others investigate the impact of lightning induced TLE disturbances on the conductivity profile in Earth's mesosphere by tracking man-made radio signals.

The idea that lightning affects the signature of radio emission has also been put forward by Schellart et al. [2015]. Schellart et al. demonstrated that terrestrial lightning leaves a fingerprint on the radio emission from cosmic ray induced air showers. Vorgul and Helling [2016] developed the mathematical framework for the effect of extraterrestrial lightning on coherent radio emission on brown dwarfs: They developed a general model to describe 


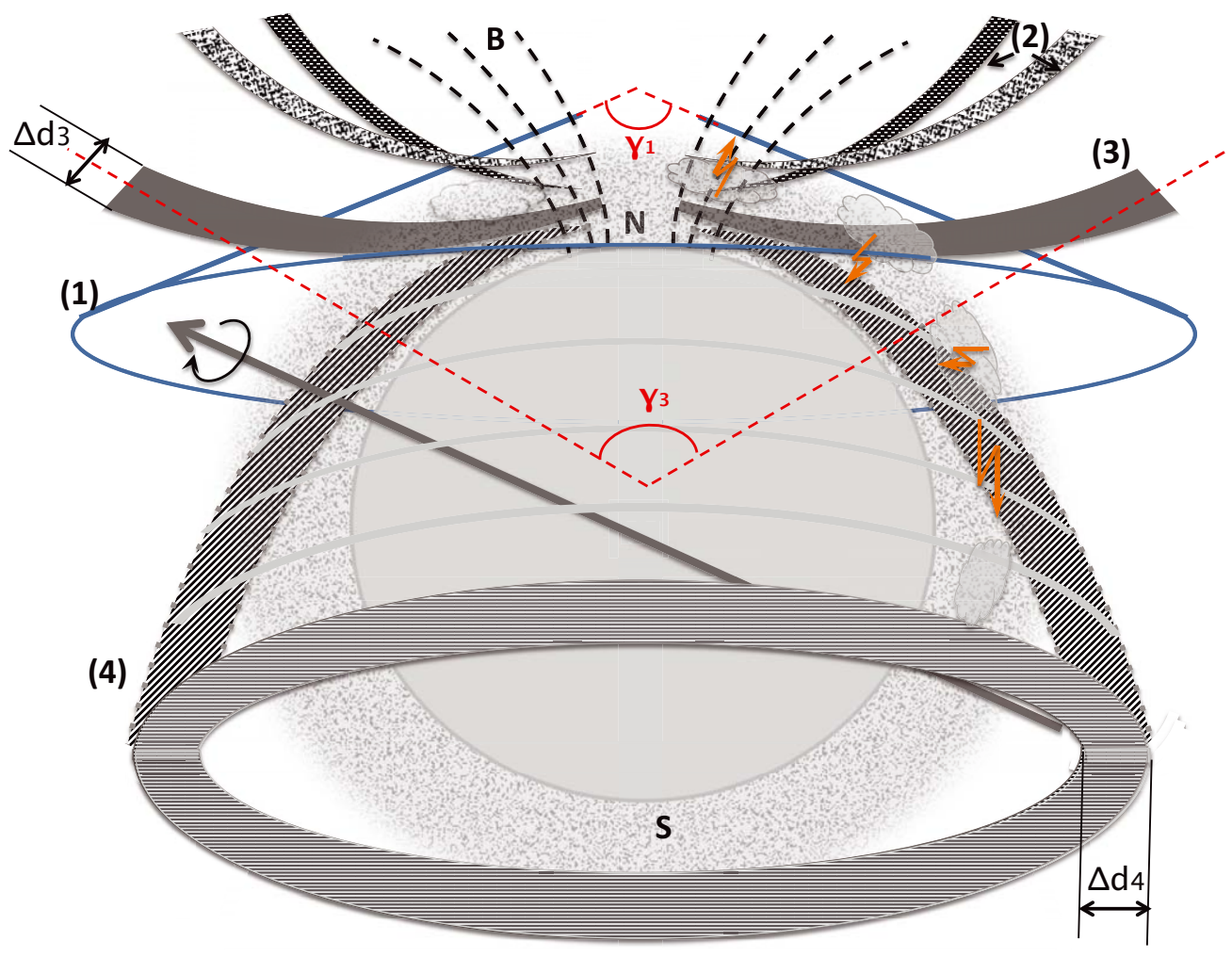

Figure 3: Coherent (radio) emission interacting with a cloudy atmosphere where lightning occurs. Depending on the source area of the coherent emission near the magnetic pole, the emission cones $(1-4)$ have different opening angles $(\gamma)$. The beam will interact more $\left(\Delta d_{4}\right)$ or less $(\Delta d$ for one of cones 2) with the ambient medium, and even beam crossing may occur (2) [from Vorgul and Helling, 2016].

the influence of flash ionization events (like lightning or expositions of similar intensity) on pre-existing radiation that passes through the region where the transient events happen. A (direct) problem of electromagnetic field transformation was solved as a result of the flash transient events and have provided a recipe for observers in Section 5.5 of Vorgul and Helling [2016]. The main findings are:

(a) The transformation of coherent emission by fast (compared to hydrodynamic processes in the atmosphere) transient processes in the medium of its propagation can be observable.

(b) Attributing larger signal perturbations to more powerful processes is not always correct, as more powerful processes can lead to smaller response signals.

(c) Flash ionization processes of intermediate intensity can be detected by short-term amplification of the original, unperturbed signal. The response field's duration can be related to the flash ionization's timescale and intensity.

(d) If the initial emission signal is powerful enough to detect the character of damping, small pulse's amplitude and duration in the response field can provide information about the ionization flash magnitude (peak value of conductivity) and duration.

(e) If the detection of the electromagnetic field's variation is possible for several related 
flashes (like multiple strokes of the same lightning discharge) and allows deriving the conductivity peak values from additional information, it would be possible to determine whether the discharges started as runaway electron break-down or rather like capacitor discharges. This can be done by deriving the dependence of the field responses' maximum values on the maximum conductivity change. The absence of a noticeable plateau in this dependence [see Figure 5 in Vorgul and Helling, 2016] suggests that the runaway breakdown discharge is likely to be a source of the ionization, which requires about 10 times lower voltage to enable the discharge in comparison to a conventional capacitor-like discharge. If the plateau is noticeable, then a capacitor-like discharge is more probable.

\section{Conclusion}

Extrasolar planets and brown dwarfs are exposed to a variety of ionizing processes, externally, globally and locally. Depending on their location in the galaxy (star forming region vs. galactic bulge) but also on the kind of host star or companions, their atmospheres will be more or less dynamic or chemically altered by plasma processes. Both object classes (exoplanets and brown dwarfs) do form clouds that can be very different, chemically and dynamically, from what we know from Earth or in the solar system. While this results in different cloud particles sizes and material compositions, the basic processes of lightning initiation and propagation are fundamentally the same. We therefore can apply concepts developed on Earth in order to study lightning occurring in extraterrestrial planets. Predicting spectral fingerprints will, however, require the modeling of the actual gas composition which can be very different from Earth.

Acknowledgments. Ch. H. and I. V. highlight the financial support of the European community under the FP7 ERC starting grant 257431. Most of the literature search has been performed using ADS. The Editors thank Yoav Yair and two anonymous reviewers for their help in evaluating this paper.

\section{References}

Ardaseva, A., P. B. Rimmer, I. Waldmann, M. Rocchetto, S. N. Yurchenko, Ch. Helling, and J. Tennyson, Lightning chemistry on Earth-like exoplanets, MNRAS, 470, 187196, 2017.

Burinskaya, T.M., and M. M. Shevelev, Generation of auroral kilometric radiation in inhomogeneous magnetospheric plasma, Geomagnetism and Aeronomy, 57, 16-23, 2017.

Casewell, S. L., K. A. Lawrie, P. F. L. Maxted, M. S. Marley, J. J. Fortney, P. B. Rimmer, S. P. Littlefair, G. Wynn, M.R. Burleigh, and Ch. Helling, Multiwaveband photometry of the irradiated brown dwarf WD0137-349B, MNRAS, 447, 4, 3218-3226, 2015 .

Cairns, R. A., I. Vorgul, and R. Bingham, Cyclotron maser radiation from an inhomogeneous plasma, Physical Review Letters, 101, id.215003, 2008. 
Cairns, R. A., I. Vorgul, R. Bingham, K. Ronald, D. C. Speirs, S. L. McConville, K. M. Gillespie, R. Bryson, A. D. R. Phelps, B. J. Kellett, A. W. Cross, C. W. Roberston, C. G. Whyte, and W. He, Cyclotron maser radiation from inhomogeneous plasmas, Physics of Plasmas, 18, id.022902, 2011.

Gray, M., Maser Sources in Astrophysics, Cambridge University Press, Cambridge, 2012.

Haldoupis, C., T. Neubert, U. S. Inan, A. Mika, T. H. Allin, and R. A. Marshall, Subionospheric early VLF signal perturbations observed in one-to-one association with sprites, J. Geophys. Res., 109, A10303, 2004.

Hallinan, G., A. Antonova, J. G. Doyle, S. Bourke, W. F. Brisken, and A. Golden, Rotational modulation of the radio emission from the M9 dwarf TVLM 513-46546: Broadband coherent emission at the substellar boundary? ApJ, 653, 690-699, 2006.

Hallinan, G., A. Antonova, J. G. Doyle, S. Bourke, C. Lane, and A. Golden, Confirmation of the electron cyclotron maser instability as the dominant source of radio emission from very low mass stars and brown dwarfs, ApJ, 684, 644-653, 2008.

Hallinan, G., S. P. Littlefair, G. Cotter, S. Bourke, L. K. Harding, J. S. Pineda, R. P. Butler, A. Golden, G. Basri, J. G. Doyle, M. M. Kao, S. V. Berdyugina, A. Kuznetsov, M. P. Rupen, and A. Antonova, Magnetospherically driven optical and radio aurorae at the end of the stellar main sequence, Nature, 523, 568-571, 2015.

Helling, Ch., P. Woitke, and W.-F. Thi, Dust in brown dwarfs and extra-solar planets. I. Chemical composition and spectral appearance of quasi-static cloud layers, Astron. Astrophys., 485, 547, 2008.

Helling, Ch., P. B. Rimmer, I. M. Rodriguez-Barrera, K. Wood, G. B. Robertson, and C. R. Stark, Ionisation and discharge in cloud-forming atmospheres of brown dwarfs and extrasolar planets, Plasma Phys. and Controlled Fusion, 58, id.074003, 2016a.

Helling, Ch., R. G. Harrison, F. Honary, D. A. Diver, K. Aplin, I. Dobbs-Dixon, U. Ebert, S. Inutsuka, F. J. Gordillo-Vazquez, and S. Littlefair, Atmospheric electrification in dusty, reactive gases in the solar system and beyond, Surv. in Geophys., 37, 4, 705-756, 2016b.

Hodosán, G., P. B. Rimmer, and Ch. Helling, Is lightning a possible source of the radio emission on HAT-P-11b? MNRAS, 461, 1222-1226, 2016.

Hodosán, G., Ch. Helling, and P.B. Rimmer, Exo-lightning radio emission: The case study of HAT-P-11b, in Planetary Radio Emissions VIII, edited by G. Fischer, G. Mann, M. Panchenko, and P. Zarka, Austrian Academy of Sciences Press, Vienna, 345-356, 2017.

Inan, U.S., S. A. Cummer, and R. A. Marshall, A survey of ELF and VLF research on lightning-ionosphere interactions and causative discharges, J. Geophys. Res., 115, A00E36, 2010.

Lee, G., I. Dobbs-Dixon, Ch. Helling, K. Bognar, and P. Woitke, Dynamic mineral clouds on HD 189733b. I. 3D RHD with kinetic, non-equilibrium cloud formation, Astron. Astrophys., 594, id.A48, 24 pp., 2016. 
Longstaff, E. S., S. L. Casewell, G. A. Wynn, P. F. L. Maxted, and Ch. Helling, Emission lines in the atmosphere of the irradiated brown dwarf WD0137-349B, MNRAS, 471, 1728-1736, 2017.

Mallios, S.A., and V.P. Pasko, Charge transfer to the ionosphere and to the ground during thunderstorms, J. Geophys. Res., 117, A08303, 2012.

Nikolov, N., D. K. Sing, N. P. Gibson, J. J. Fortney, T. M. Evans, J. K. Barstow, T. Kataria, and P.A. Wilson, VLT FORS2 comparative transmission spectroscopy: Detection of $\mathrm{Na}$ in the atmosphere of WASP-39b from the ground, Astrophys. J., 832, id.191, 9 pp., 2016.

Pritchett P. L., R. Strangeway, R. Ergun, and C. Carlson, Generation and propagation of cyclotron maser emissions in the finite auroral kilometric radiation source cavity, J. Geophys. Res., 107, A12, 2002.

Rimmer, P. B., Ch. Helling, and C. Bilger, The influence of galactic cosmic rays on ionneutral hydrocarbon chemistry in the upper atmospheres of free-floating exoplanets, Int. J. Astrobiology, 13, 173-181, 2014.

Rodriguez-Barrera, M. I., Ch. Helling, C. R. Stark, and A. M. Rice, Reference study to characterize plasma and magnetic properties of ultracool atmospheres, MNRAS, 454, 3977-3995, 2015.

Rodriguez-Barrera, M. I., Ionisation in atmospheres of brown dwarfs ad giant gas planets, PhD Thesis, University of St. Andrews, UK, 2016.

Schmidt, S. J., S. L. Hawley, A. A. West, J. J. Bochanski, J. R. A. Davenport, J. Ge, and D. P. Schneider, BOSS ultracool dwarfs. I. Colors and magnetic activity of $\mathrm{M}$ and L dwarfs, Astron. J., 149, id.158, 21 pp., 2015.

Schellart, P., et al. (73 co-authors), Probing atmospheric electric fields in thunderstorms through radio emission from cosmic-ray-induced air showers, Phys. Rev. Lett., 114, id.165001, 2015.

Sing, D., J. J. Fortney, N. Nikolov, H. R. Wakeford, T. Kataria, T. M. Evans, S. Aigrain, G. E. Ballester, A. S. Burrows, D. Deming, J.-M. Désert, N. P. Gibson, G. W. Henry, C. M. Huitson, H. A. Knutson, A. Lecavelier Des Etangs, F. Pont, A. P. Showman, A. Vidal-Madjar, M. H. Williamson, and P. A. Wilson, A continuum from clear to cloudy hot-Jupiter exoplanets without primordial water depletion, Nature, 529, 59-62, 2016.

Vorgul, I., and Ch. Helling, Flash ionization signature in coherent cyclotron emission from brown dwarfs, MNRAS, 458, 1041-1056, 2016.

Williams, P. K. G., S. L. Casewell, C. R. Stark, S. P. Littlefair, Ch. Helling, and E. Berger, The first millimeter detection of a non-accreting ultracool dwarf, MNRAS, 815, id.64, 6 pp., 2015. 
